\title{
DAS VERHALTTIS DER VEREINIGTEN STAATEN ZU LATEINAMERIKA
}

\author{
KNUD KRAKAU
}

Es wäre unrealistisch anzunehmen, man könnte im Rahmen eines kurzen Papiers ${ }^{1}$ dieser Thematik gerecht werden, gleich wie man sie anpackt. Sie ist in ihrer historischen Dimension ebenso komplex wie in ihrer gegenwärtigen Struktur. Das beginnt mit dem Sammelbegriff "Lateinamerika": Er ist im Grunde nur der hilflose Versuch, mittels einer geographischen Kategorie zu einer Einheit zusammenzufassen, was sich durch Vielfalt und Heterogenität auszeichnet - Vielfalt der Kulturen, Wirtschaften, Interessen etc. Es gibt heute mindestens so viele US-amerikanische Politiken gegenüber Lateinamerika wie es dort selbständige politische Einheiten gibt - vermutlich noch mehr. Denn State Department, White House, Defense Department usw. verfolgen oft genug schlecht koordinierte bis widersprüchliche Politiken. Deshalb sollen im folgenden einige allgemeine Aspekte dieser Beziehungen skizziert und diese allgemeinen Linien dann auf einige Punkte hin konzentriert werden.

\section{Historische Vorbelastungen}

Ein herausragendes Merkmal in der Konzipierung und Handhabung aller staatlichen politischen Beziehungen der USA zu Lateinamerika scheint ein Realitätsdefizit zu sein. Es zieht sich wie ein roter Faden durch die über 150jährige Geschichte dieser Beziehungen. Das schließt natürlich sehr harte „reale“ politische Maßnahmen nicht aus: Konflikte, Interventionen, z. B. zum Schutze von Investitionen, strategischen Positionen usw. Wir beziehen uns vielmehr auf den konzeptuellen Rahmen der amerikanischen Politik, der den Charakter und das Ziel von Einzelentscheidungen präformiert, wo nicht determiniert. Die USA haben seit 1810 an einer Perzeptionsverzerrung gegenüber den lateinamerikanischen Realitäten gelitten, und - um das Ergebnis vorwegzunehmen - die gegenwärtige Phase dieser Beziehungen ist dadurch gekennzeichnet, daß objektive Veränderungen die USA zwingen, diese Verzerrungen zurechtzurücken, daß aber dieser Lernprozeß mühsam und langwierig ist. Diese Perzeptionsverzerrung findet ihren wichtigsten Ausdruck in dem stark ideologisierten Konzept der "Westlichen Hemisphäre" — „The Western Hemisphere Idea“2. Die Vereinigten Staaten entwickelten nach der Unabhängigkeit Lateinamerikas im 19. Jahrhundert allmählich dieses Konzept einer „special hemispheric community“. Sie beruhte ihrer Vorstellung zufolge auf gemeinschaftlichen neuen, aufgeklärten und fortschrittlichen Werten von Politik, Kultur und Humanität; sie unterlag "neuen Gesetzen“ im Verhältnis ihrer Glieder zueinander wie zwischen ihnen insgesamt und der übrigen Welt. Sie gab den Anstoß z. B. zu den Bemühungen um ein sog. spezifisch „amerikanisches Völkerrecht" ${ }^{\text {"3. Das }}$

\footnotetext{
1 Ihm liegt ein Vortrag zugrunde, den der Verfasser im Mai 1977 auf der Tagung des Arbeitskreises für überseeische Verfassungsvergleichung gehalten hat. Der Text wurde leicht überarbeitet und um den wissen-

2 Vgl. hierzu stellvertretend für eine breite Strömung in der nordamerikanischen Literatur über Lateinamerika Arthur P. Whitaker, The Western Hemisphere idea. Its rise and decline (Ithaca N. Y. 1954); Wilfrid H. Callcott, The Western Hemisphere. Its influence on United States policies to the end of World War II (Austin, London 1968).

3 So z. B. in den ersten Jahrzehnten dieses Jahrhunderts in den zahlreichen Werken des chilenischen Völkerrechtlers Alejandro Alvarez u. a. lateinamerikanischer Juristen.
} 
Dilemma lag darin, daß das Konzept der „Westlichen Hemisphäre“ eine „natürliche Harmonie" der Interessen und Anschauungen Nord- und Südamerikas postulierte, tatsächlich aber ganz wesentlich auf Projektionen nordamerikanischer Erfahrungen und Interessen beruhte und von den USA definiert wurde. Es überspielte die tiefgreifenden kulturellen, ethnischen, sozioökonomisch-politischen Unterschiede zwischen den USA und Lateinamerika rhetorisch-ideologisch oder verdrängte sie ganz real aus dem nordamerikanischen Bewußtsein.

a) So interpretierte man in den USA die Unabhängigkeitsbewegungen und nachfolgenden Staatsgründungen in Lateinamerika als Fortsetzung und Übernahme des nordamerikanischen Modells des republikanischen, freiheitlich-demokratischen Verfassungsstaates. Dieser war ja seinerseits als qualitativ höhere Stufe auf der menschlischen Fortschrittsleiter erschienen. Man übersah (und übersieht) die Andersartigkeit der politischen Kulturen, der sozialen, ethnischen, ökonomischen Strukturen.

b) Ein geopolitischer Determinismus schien - so nahmen schon Jefferson, J. Q. Adams und andere frühe politische Autoritäten an ${ }^{4}-$ den Süden, insbesondere Mittelamerika und die Karibik, nach politischen "Gravitationsgesetzen“ den Vereinigten Staaten zuzuführen. Man dachte allerdings nicht unbedingt an ein neues Kolonialverhältnis, sondern eher an die gleichberechtigte Integration nach dem Vorbild der Northwest Ordinance 1787, die allgemein der Aufnahme der kontinentalen Territorien in die Union zugrundegelegt wurde. Diese Erwartungen erfüllten sich zwar in der Geschichte nicht - eben weil sie falsch gegründet waren. Dennoch entwickelte sich aus beiden Umständen zusammen die Vorstellung der im Gegensatz zu Europa größeren „Nähe“ Lateinamerikas zu den USA. Die tatsächliche Situation ist sogar im geographischen Sinne weitgehend, erst recht im kulturellen und politischen Sinne genau umgekehrt.

c) Schließlich spielten Handel und Wirtschaft eine bedeutende Rolle. Schon zur Kolonialzeit ging der relative Wohlstand der nordamerikanischen Kolonien zu einem erheblichen Teil auf den Handel mit Westindien zurück. Dieser Handel weitete sich im 19. Jahrhundert in Konkurrenz zu England auf ganz Lateinamerika aus. Die größte Bedeutung erlangte Lateinamerika wirtschaftlich für die USA (und umgekehrt USA für die Wirtschaften Lateinamerikas) im Zusammenhang mit der sich beschleunigenden Massenindustrialisierung der USA nach 1870-80. Insgesamt war Lateinamerika für die USA, wie neuere Untersuchungen zeigen, weniger wichtig als Markt für die nordamerikanische industrielle Produktion. Diese Rolle des Marktes für die USA spielte im wachsenden Maße Europa. Lateinamerika fungierte insbesondere als Rohstofflieferant für die nordamerikanische Industrie. Wenige Zahlen mögen das verdeutlichen. So gingen 1900 drei Viertel aller USamerikanischen Exporte nach Europa - weniger als 1/10 nach Lateinamerika -, 1910 waren es zwei Drittel. Für die letzten Jahrzehnte nach 1945 ist dieser allgemeine Trend im Verhältnis der industrialisierten Staaten untereinander im Gegensatz zur Nord-Süd-Relation bekannt. Die US-amerikanischen Importe aus Lateinamerika, bestehend vor allem aus Rohstoffen und Nahrungsmitteln, verdoppelten sich jeweils im Zeitraum 1880-1900-1910 und machten zum letztgenannten Zeitpunkt ein Drittel aller nordamerikanischen Importe aus (1900: 1/5). Von Latein-

4 Vgl. Jefferson, zit. bei Callcott, a. a. O. S. 15; Adams: „Cuba ... can gravitate only towards the North American Union, which by the same law of nature cannot cast her off from its bosom." In: W. C. Ford ed.: The writings of John Quincy Adams (New York 1917) Bd. 7, S. 373. 
amerika aus gesehen repräsentierten die Exporte in die USA im Jahre 1913 für vier Länder (Chile, Mexiko, Peru, Bolivien) 43 Prozent aller ihrer Exporte und für vier andere Länder (Brasilien, Kolumbien, Kuba, Ekuador) sogar 48 Prozent ${ }^{5}$. Der hieraus resultierende enorme direkte und indirekte Einfluß der USA auf Wirtschaft, Politik, Gesellschaft und Identitätsbewußtsein Lateinamerikas braucht nicht besonders hervorgehoben $\mathrm{zu}$ werden. Er spiegelt sich noch in dem wichtigsten theoretischen Beitrag Lateinamerikas der letzten Jahre zur analytischen Erfassung dieser Beziehungen: der Entwicklung der dependencia-Theorie ${ }^{6}$ als Abwehrhaltung oder Strategie zur Identitätsbewahrung (oder -gewinnung).

Betont werden muß aber, daß die USA noch immer ein unterentwickeltes Bewußtsein dieser Macht-Relation zu Lateinamerika haben, obwohl sie doch selbst noch lange nach 1776/1783 an genau derselben Dependenz-Rolle im Verhältnis zu England gelitten hatten. Die Gründe u. a.: Handel/Wirtschaft implizieren „covert forms of power", die neben der klassischen "overt" Form der militärisch-politischen Macht ${ }^{7}$ nicht als solche gesehen werden; Handel/Wirtschaft galten - Erbe der Aufklärung, das eben wegen seiner Wirksamkeit später um so leichter klassische rationalisierende Ideologie-Funktion übernehmen konnte - als schlechthin rationale Verhaltensweisen: human, aufgeklärt, fortschrittlich im Gegensatz zur irrationalen traditionellen Macht-Politik. Schon im 18. Jahrhundert war man sich durchaus bewußt, daß auch Handels- und Wirtschaftspolitik „Politik“ waren. Gleichzeitig konnte man aber, im Glauben an die Rationalität wirtschaftenden Verhaltens, das den größeren gemeinsamen Nutzen aller verbürge, überzeugt sein, die „Politik“ (als irrationale Ausübung von Macht) durch rationale, d. h. vernünftige, moralische, fortschrittliche Verhaltensmodi zu ersetzen ${ }^{8}$. Handlungen und Werte, die dieser Sphäre zuzuordnen sind, erscheinen nicht zuletzt deshalb noch heute ausgesprochen positiv besetzt. Die Amerikaner verstellen sich damit den Blick für die Lage derer, die wie die Lateinamerikaner von der „Vernünftigkeit“ der Verhältnisse nach Lage der Dinge entschieden weniger begünstigt werden.

Um zu wiederholen: Obwohl das „Westliche-Hemisphäre“-Konzept in der angedeuteten Weise auf die Projektion der Erfahrungen und die Rationalisierung der Interessen der Vereinigten Staaten zurückgeht und sie es definieren, leiten sie dennoch zugleich normative Wirkungen für die interamerikanischen Verhältnisse aus $\mathrm{ihm}$ ab. Diese finden ihren zusammenfassenden Ausdruck in der Monroe-Doktrin: die vermeintlichen politisch-ideologischen Gemeinsamkeiten der „Westlichen Hemisphäre", deren Absetzung gegen Europa und der zu ihrem Schutze vor Europa erhobene Abwehranspruch der Vereinigten Staaten'. Obwohl ursprünglich keine imperiale, sondern eine defensive Strategie für das Schutzgut „Westliche Hemisphäre", schlägt die Monroe-Doktrin bekanntlich in eine hegemoniale Strategie um: Eine von den Vereinigten Staaten postulierte, tatsächlich aber nicht oder nur in

5 Nach Gabriel Kolko: Main currents in modern American history (New York usw. 1976), S. 37 f.

$6 \mathrm{Vgl}$. dazu außer den bekannten Arbeiten von Frank, Furtado, Stavenhagen, Sunkel u. a. z. B. die Bibliographie mit ausschließlich lateinamerikanischen Beiträgen dazu bei Dieter Senghaas (Hrsg.): Imperialismus und strukturelle Gewalt. Analysen über die abhängige Reproduktion (Frankfurt 1972) S. $390-399$.

7 Diese Unterscheidung geht auf Reinhold Niebuhr zurück.

8 Das u. a. ist das Thema von Gerald Stourzh: Benjamin Franklin and American foreign policy (Chicago 1954), passim, bes. Kap. IV, S. $113 \mathrm{ff}$.

9 Es sei darauf verzichtet, von den ganze Bibliotheken füllenden Büchern zur Monroe-Doktrin auch nur ein einziges zu erwähnen. Klargestellt soll aber sein, daß die Monroe-Doktrin nur die Symbol gewordene Kurzformel für eine Haltung ist, die lange vor 1823 bestand und noch heute aktuell ist. Jefferson über Rebellen in Kuba und Mexiko: "We consider their interests and ours as the same, and that the object of both must be to exclude all European influence from this hemisphere ${ }^{\alpha}$, zit. nach Callcott, a. a. O. S. 15 . 
engen Grenzen vorhandene politisch-moralische (ideologische) Gemeinsamkeit wird von den USA einseitig gegen außen "verteidigt“.

Das aber ist das Dilemma der Monroe-Doktrin oder der Lateinamerika-Politik der USA schlechthin:

a) Im Namen ihres Schutzgutes, der „Westlichen Hemisphäre“, haben die Vereinigten Staaten - um sie „schützen“ zu können - auf die inneren Verhältnisse der Staaten Lateinamerikas durch direkte oder indirekte Interventionen Einfluß $\mathrm{zu}$ nehmen gesucht, um die politisch-ideologischen Gemeinsamkeiten ggfs. überhaupt erst herzustellen. $\mathrm{Zu}$ diesen Basis-Zielen gehören: Wahlen überhaupt oder doch „demokratische“ Wahlen, Bildung „verfassungsmäßiger" Regierungen, Beachtung „des internationalen Rechts“, Schaffung eines „günstigen Investitionsklimas“, Enteignungsentschädigungen, neuerdings: Beachtung der Menschenrechte. Die Mittel sind von jeher: politischer Druck bis zu militärischen Interventionen, Belohnungen - Strafen: Gewährung oder Versagen der Anerkennung neuer Regierungen, von Krediten, Auslandshilfe usw.

b) Darüber hinaus haben die Vereinigten Staaten zumindest dann, wenn ihrer Meinung nach außerkontinentale Einflüsse im Spiele waren, auch die internationalen Beziehungen der lateinamerikanischen Staaten inter se (Stichwort „Friedenswahrung“ und „Export der Revolution“) und schließlich

c) auch im Verhältnis zur nichtamerikanischen Welt zu kontrollieren gesucht. Hier ist an die Venezuela-Krise 1902/3 oder die Ereignisse im und nach dem 2. Weltkrieg zu erinnern, insbesondere im Zusammenhang mit dem „internationalen Kommunismus" in Guatemala, Kuba usw.

Die Reaktion Lateinamerikas schwankte seit 1823 zwischen nationalistischem Protest und partieller Zustimmung dort, wo die Existenz von Herrschaftseliten an die Vereinigten Staaten gebunden war oder eine wirkliche oder vermeintliche extrakontinentale Bedrohung im Spiele war.

In jahrzehntelangen Bemühungen gelang es insbesondere seit 1933, dieses prekäre Verhältnis im „Interamerikanischen System“, später der OAS, zu institutionalisieren. Den Staaten Lateinamerikas ging es dabei in erster Linie darum, mit Hilfe dieses neuen Instrumentes wenigstens die Chance einer Respektierung ihrer Souveränität und Integrität sichern zu helfen. Ihr Maximalziel war der nordamerikanische Verzicht auf das bis dahin beanspruchte Interventions- „Recht“. Die USA hingegen suchten die kollektive Anerkennung des „Westliche-Hemisphäre“-Konzeptes, und zwar seiner politisch-ideologischen Basis-Aspekte im weiteren OAS-System, der defensiven Monroe-Aspekte insbesondere im Rahmen des Rio-Vertrages. Eine solche zumindest verbale Homogenität war allenfalls in der Hoch-Zeit des Kalten Krieges im Kontext des Anti-Kommunismus herstellbar. In Caracas 1954 konnten die Vereinigten Staaten mit Befriedigung das einstimmige Bekenntnis Lateinamerikas zu einer kollektiven Monroe-Doktrin auf der Basis des „Westliche-Hemisphäre“Konzeptes protokollieren. Sie bewährte sich noch einmal in den kollektiven Sanktionsbeschlüssen gegen Kuba 1964.

Daneben aber haben die USA stets an ihrer klassischen einseitigen Monroe-Politik festgehalten: d. h. einseitig und allein zu definieren, zu urteilen und zu handeln. Sie argumentieren dabei (Kuba 1961, 1962, Dominikanische Republik 1965) stets etwa wie folgt: das Schutzgut "Westliche Hemisphäre“ und das Vorgehen zu seinem Schutz sind zwar kollektiv definiert. Wenn aber das zur Gefahrenabwehr ihrer Ansicht nach „Erforderliche“ nicht kollektiv getan wird, müssen die Vereinigten 
Staaten hilfsweise, stellvertretend, in Geschäftsführung ohne Auftrag o. ä. — aber eben allein für die "community" handeln. Sie akzeptieren nicht, daß erst auch die Gemeinschaftlichkeit des Beschlusses und seiner Exekution dessen interamerikanische Systemkonformität garantiert ${ }^{10}$. Oder sie interpretieren wie 1965 in der Dominikanischen Republik a) die bloße Vermutung einer Bedrohung der Schutzgüter der "Westlichen Hemisphäre" durch bestenfalls denkbare, keineswegs vollzogene revolutionäre Veränderungen zugleich automatisch b) als extrakontinentale „threat of intervention" durch „subversive forces known as international communism" (Repräsentantenhaus), die im Sinne der Monroe-Doktrin auch gewaltsame Abwehrmaßnahmen rechtfertige. Die USA behielten sich also jedenfalls auf dem Höhepunkt ihrer Verunsicherung durch diese Vorgänge, aber andeutungsweise auch noch in der jüngsten Vergangenheit (Kissinger) vor, jede Abweichung von der Orthodoxie der Ideologie der "Westlichen Hemisphäre“ als direkte, und zwar außeramerikanische Bedrohung ihrer eigenen Sicherheit zu interpretieren, die den Abwehrreflex der Monroe-Doktrin bis zu „defensiver Gewalt“ wenn möglich kollektiv, wo nötig unilateral auslösen würde ${ }^{11}$.

Die USA leiten also von einem „community“-Konzept, das sie weitgehend selbst entwickelt haben und das nur partielle, d. h. gruppenspezifische und selbst dann meist rein verbale Zustimmung in Lateinamerika findet, permissive und normative Konsequenzen her. Daraus entwickelte sich der permanente Widerspruch zwischen Anspruch und Rhetorik einerseits und der historisch-politischen Realität andererseits - das eingangs hervorgehobene Realitätsdefizit in der nordamerikanischen Haltung gegenüber Lateinamerika. Immer wieder suchten und suchen die USA den Widerspruch rein verbal - oder dadurch aufzuheben, daß sie als Hegemon handeln. Für den ersteren Ansatz gab H. Kissinger, nicht gerade bekannt als Polit-Lyriker, kurz vor Ende seiner Amtszeit noch ein Beispiel. Auf der OAS-Konferenz vom Juni 1976 in Santiago/Chile führte er aus: „This hemisphere is unique; there is no other grouping like it in the world. We have indeed a special relationship ...". Und etwas später: „Nothing has been more central to our hopes than the relationships of the nations of this hemisphere. They are a priceless foundation of past achievement, a vital and progressing process of present cooperation, and our brightest vision for the future... We regard the concerns of this hemisphere as our first priority ... shared vision ... common heritage"; noch heute seien Amerikaner "not European. Our traditions and institutions have something new in them ... This hemisphere is the world's laboratory of human freedom, the just and ultimate refuge of the rights of $\operatorname{man}^{12}$." - usw. usw. Diese inflationierte Rhetorik klingt jedenfalls Ende 1976 peinlich, hohl und unglaubwürdig. Sie beeinträchtigt nicht nur die Kommunikation mit Lateinamerika. Vor allem verstellt sie den Vereinigten Staaten selbst die Einsicht in das Notwendige: nicht besondere, sondern schlechthin normale, allgemeine Beziehungen zu den Staaten Lateinamerikas zu unterhalten; ferner die Bereitschaft, sie in ihren inneren Verhältnissen wie äußeren Beziehungen untereinander und insbesondere zur nichtamerikanischen Welt als Staaten prinzipiell gleichen Rechtes zu behandeln.

10 Als Beispiel die offizielle amerikanische Rechtfertigung der Intervention in der Dominikanischen Republik 1965 durch den Legal Adviser des Dept. of State L. Meeker: The Dominican situation in the perspective of international law, in: Department of State Bulletin 53 (1965 II) S. 60-65.

$11 \mathrm{Zit}$. des Repr. Hs. bei Abraham F. Lowenthal: The United States and Latin America: ending the hegemonic presumption, in: Foreign Affairs 55 (1976/77), S. 199-213, 205. - Vgl. Kissinger, Rede am 6. 7. 1976, USIS Official Text v. 7. 7. 1976, S. 9. der das „long held (a) common interest in shielding our hemisphere from the intrusion of others ${ }^{\alpha}$ betonte.

12 In: Dept. State Bulletin 75 (1976 II) S. 10-11; das 2. Zit. aus einer Rede vor lateinamerikanischen UNDelegierten am 7. 10. 1976, ibid. S. 555-558. 
Die „Westliche Hemisphäre" als „community“ - was immer ihre (Pseudo-)Realität in der Vergangenheit gewesen sein mag - zerbröckelt in der Gegenwart mehr und mehr. Demselben Erosionsprozeß unterliegt ihre permissiv-normative Legitimationsbasis für die nordamerikanische Hegemonialrolle. Im selben Maße zerfällt auch das interamerikanische System der OAS. Das liegt u. a. an der Auflockerung der globalen Konfliktfronten, nicht zuletzt aber auch daran, daß auf beiden Seiten (Nord- und Südamerika) die Überzeugung verlorengegangen oder verlorenzugehen im Begriff ist, daß die USA für die wichtigsten lateinamerikanischen Erwartungen noch einen konkreten Beitrag leisten oder (nach den "Grenzen des Wachstums") auch nur ein relevantes Modell anbieten könnten: nämlich für das Verlangen nach wirtschaftlicher Entwicklung und politisch-sozialer Veränderung in Richtung auf mehr Gerechtigkeit, Gleichheit, Partizipation. Carters Re-Moralisierung der amerikanischen (Außen-)Politik entspringt vielleicht gerade der Einsicht in dieses Unvermögen. - Dieser allgemeine Erosionsprozeß sei an einigen Gegenwartsproblemen näher erläutert.

\section{Die Gegenwart: Menschenrechte und die Tradition der Moralhaftigkeit in der amerikanischen Politik}

Die gegenwärtige Menschenrechts-Politik Carters - sofern man sein „almost theological commitment" (NY Times) „to restore the moral authority of this country in its conduct of foreign policy“ (Carter) eine "Politik“ nennen kann unterscheidet sich strukturell wenig von früheren Politiken zur Durchsetzung politisch-moralischer Prinzipien, die alle in der amerikanischen Tradition verankert sind. Carter und seine Administration werden nicht müde, bzgl. der Menschenrechte immer wieder auf diese Tradition hinzuweisen. Gleichwohl versteht die Carter-Administration ihre Menschenrechtsakzentuierung als „beginning of a new influence for ideas and ideals in the world ${ }^{13}$." Eine derartige bewußte Prinzipienund Moralhaftigkeit ist allerdings in liberalen Administrationen (F. D. R., Kennedy, Johnson) im allgemeinen stärker ausgeprägt als in republikanischen, die dazu neigen, Lateinamerika den wirtschaftlichen Impulsen und Interessen zu überlassen und dann für eine Politik (kurzfristiger) Stabilität und ein "günstiges Investitionsklima“ einzutreten. Auch die demokratischen Regierungen verstehen ihre Ziele natürlich "politisch": sie sehen Stabilität längerfristig in Kategorien demokratischfreiheitlicher Legitimität. „Die Menschenrechte“ gehören - ähnlich wie bei Kennedy demokratische Wahlen, sozialer und wirtschaftlicher Wandel (Agrar-Steuerreformen, „Allianz für den Fortschritt") - zum Kernbereich US-amerikanischen Selbstverständnisses wie auch zum rhetorisch allseits akzeptierten Repertoire politisch-humaner Werte in ganz Amerika. Über die Überzeugung hinaus, daß „words are action" (Carter), werden früher wie heute die gleichen Sanktionen (Belohnungen - Strafen) angewandt: Gewährung oder Versagung der Anerkennung neuer Regierungen (Kennedy, Johnson), von Wirtschafts-, Militär- und technischer Hilfe (Kennedy, Carter) ${ }^{14}$, von Ausbildungshilfen für Militär und Polizei, des Zuganges

13 Stellvertr. Außenminister W. Christopher, Rede am 17. 6. 1977 in Pittsburgh, USIS News Report v.

14 W. Christopher nennt diese, „tools“ der US-Außenpolitik ausdrücklich so beim Namen u. ergänzt sie lediglich um "diplomatic approaches" oder wo nötig "public comment" i. S. öffentlicher Verurteilung und Anrufung der "öffentlichen Weltmeinung “ "jedenfalls "... we shall never apologize for expressing our commitment to our principles". Rede am 10. 8. 1977, USIS News Report. Ebenso Secr. of State Vance, folgende Anm. 
zum amerikanischen Zuckermarkt, von Zollpräferenzen usw. Die Carter-Administration erwägt ferner, die Menschenrechtsfrage zum Kriterium für den Abschluß anderer langfristiger Verträge zu machen. In entsprechender Weise will sie ihren Einfluß in den multilateralen Kreditinstituten (Weltbank, IADB) geltend machen ${ }^{15}$. Finanzminister Blumenthal drückte das so aus: „We will instruct our representatives to these organizations to differentiate in the degree of enthusiasm with which they approve specific projects, taking human rights considerations into account ${ }^{16 \text { “" }}$

Die strukturelle Verwandtschaft der Carter'schen mit der Prinzipienpolitik seiner Vorgänger zeigt sich auch in ihren potentiellen Wirkungen: der Ubiquität des Betroffenseins oder, wie E. M. Borchard es schon vor Jahrzehnten etwas respektloser nannte, der Politik des „universal meddling“. Wie die „Unteilbarkeit des Friedens" dazu geführt hatte, daß die USA durch eine "Aggression“, eine „Friedensoder Rechtsverletzung " irgendwo in der Welt sich für unmittelbar betroffen hielten, so jetzt wieder angesichts der entsprechenden „Unteilbarkeit der Freiheit“ oder der Menschenrechte. In den Worten Carters: „Because we are free, we can never be indifferent to the fate of freedom elsewhere" (Inaugural). Seine Position wurde kürzlich von einer starken Senatsmehrheit aus beiden Parteien nachdrücklich unterstützt ${ }^{17}$. Die Frage ist hier nicht, wie weit die Internationalisierung der Menschenrechtsfrage völkerrechtlich inzwischen wirklich gediehen ist - ein bekanntlich unter Berufung auf jeweils konträre oder komplementäre Rechtsprinzipien stark umstrittenes und auch in seinen Folgewirkungen noch keineswegs voll überschaubares Problem. Wichtig ist hier, daß die amerikanische Administration eine mindestens politisch-moralische Internationalisierung als selbstverständlich zugrundelegt. Sie meint, diese sei tatsächlich von einem internationalen und - ganz besonders wichtig - nationalen Konsens getragen, und sucht damit ihre Politik international zu legitimieren. Kein UN-Mitglied, und d. h. praktisch kein Staat könne heute noch "claim that mistreatment of its citizens is solely its own business"18. Erst jetzt haben die USA als Geste guten Willens und politischer Konsequenz wenigstens die interamerikanische Menschenrechtskonvention unterzeichnet - allerdings mit geringer Aussicht auf Zustimmung des Senats.

Während nun die praktischen Folgen der amerikanischen Politik etwa gegenüber der Sowjetunion und Osteuropa rhetorisch-verbal bleiben, werden sie Lateinamerika gegenüber konkret und spürbar: Einstellung der Auslandshilfe, meist Militärhil$\mathrm{fe}$, an eine ganze Reihe von Staaten, die in den vom Foreign Assistance Act geforderten Länderberichten als schwarze Schafe in Sachen Menschenrechte erscheinen. Auch für die Wirtschafts- und Entwicklungshilfe sieht die neue Gesetzgebung des Jahres 1977 derartige Sanktionen vor ${ }^{19}$.

$15 \mathrm{Vgl}$. die allgemeinen Grundzüge in Carters großer Rede über seine Lateinamerika-Politik vor dem St.OAS-Rat am 14. 4. 1977, USIS Official Text v. 15. 4. 77. - Die grundlegende Rede von Secr. of State Vance zur Menschenrechtsfrage am 30.4.77 vor der University of Georgia, USIS News Report vom 2. 5. 77. - Daß an der Menschenrechtsdebatte mehr ihre propagandistische Verwendung als die Sache neu ist, zeigt Kissingers Rede vor der OAS General Assembly am 8. 6. 1976 in Santiago, USIS Official Text v. 9. 6. 76 .

$16 \mathrm{Am}$ 2. 4. 1977 vor dem Senate Appropriations Comm., Auszüge in: USIS Economic News v. 4. 3. 77, S. 2. Ahnlich Secr. of State Vance, vorige Anm., dort S. 5. Das Abgeordnetenhaus hatte im Sommer 1977 durch einen Zusatz in einer Haushaltsvorlage den US-Vertretern in diesen Organisationen strikt verboten, für Kredite etc. an Länder zu stimmen, die nach Ansicht des Kongresses die Menschenrechte verletzen. Der Vermittlungsausschuß hat das auf Intervention des Präsidenten (dem zumindest an Flexibilität gelegen war) dann zu einer Kann-Regelung abgeschwächt, vgl. den Bericht "Compromise, clash mark Congress' relations with Carter", USIS News Report v. 16. 8. 1977.

17 Nach International Herald Tribune (IHT) vom 28. 3. 1977, 3:1. Es hat seither viele andere Bekundungen von Zustimmung im Kongreß und in der allgemeinen Offentlichkeit gegeben.

18 Carter in seiner UN-Rede, zitiert und weitergeführt von Vance, s. o. Anm. 15.

19 Vgl. USIS News Report vom 26. 7. 1977: "Congress supports Carter initiatives on aid, human rights." 
Aber inzwischen ist in Lateinamerika ein Wandel eingetreten. Konnten die USA in den frühen 60er Jahren unter Kennedy im Hinblick auf "demokratische Wahlen“ noch gewisse „Erfolge“ erzielen, so ist die Carter-Strategie konterproduktiv:

1. Sie hat kaum Aussicht, größeren Respekt für die Menschenrechte hervorzubringen,

2. sie stärkt indirekt die Regime, die in der Gewährleistung der Menschenrechte nicht gerade ihre Hauptaufgabe sehen,

3. sie stärkt insgesamt die Region in ihren gegen die USA gerichteten oder zumindest von ihnen unabhängigen Positionen und Tendenzen, insbesondere ihre Zuordnung zur 3. Welt - damit aber das Lager jener, die den USA in dieser langfristig wohl bedeutendsten Konfliktrelation gegenüberstehen, an dem Menschenrechtsargument aber weniger Interesse haben.

Die historisch begründete Empfindlichkeit Lateinamerikas gegenüber nordamerikanischen Ingerenzversuchen ist stärker als die in demokratisch-liberalen, heute meist oppositionellen Gruppierungen durchaus vorhandene inhaltliche Übereinstimmung mit Carters Prinzipien. Anders ausgedrückt: Diese Politik führt zu einer Solidarisierung sowohl innerhalb einzelner lateinamerikanischer Staaten wie zwischen den Staaten der Region. Die relativ stabilen liberal-demokratischen Regime (Mexiko, Costa Rica, Venezuela, vielleicht noch Kolumbien) brauchen zwar die Menschenrechtsdiskussion nicht zu scheuen, müssen aber aus Überzeugung und zur Selbsterhaltung die nordamerikanische Bevormundung zurückweisen. Die Militärdiktaturen in den anderen Staaten, gleich ob in der entwicklungsprogressiven Variante (Brasilien, Panama, Peru) oder der konservativ bis reaktionären Variante (Chile, Paraguay) müssen darüber hinaus angesichts der Menschenrechtsdebatte und internationalen détente-Politik um ihre interne Stabilität und Existenz fürchten. Denn dem amerikanischen Verlangen nach "Stabilität" und anti-kommunistischer bzw. anti-Castro-Verläßlichkeit verdanken einige von ihnen Existenz und Legitimation. Sie werden durch die Carter-Prinzipien verunsichert und haben deshalb nach anfänglichen Gesten (Entlassung einiger Gefangener) ihre autoritären Strukturen noch zu festigen gesucht. Dazu war die Zurückweisung der US-amerikanischen "Zumutungen" ein wirksamer Beitrag. Außerdem sie konnten noch des Beifalls der demokratischen Opposition sicher sein.

Andererseits hat auch die neue US-Regierung das Ziel der Stabilisierung und Immunisierung Lateinamerikas gegen Einflüsse kommunistischer Staaten, insbesondere Kubas, natürlich nicht aufgegeben. Wie sie diese Widersprüche lösen will, ist bisher nicht sichtbar. Sichtbar ist aber die Protestwelle durch ganz Lateinamerika, u. a. darin, daß 7 oder 8 Staaten, darunter Argentinien, Brasilien, Chile und Uruguay, weitere Militär- und Wirtschaftshilfe der USA zurückgewiesen und entsprechende Abkommen gekündigt haben. Dabei ist nicht ohne Ironie, daß die technischen und militärischen Führungseliten dieser Staaten ihre Fähigkeiten meist an nordamerikanischen Universitäten und Militärakademien erworben haben. Der Solidarisierungseffekt wurde sicher noch verstärkt durch die öffentlichen Bestätigungen der covert activities der US-Geheimdienste wie im Chile-Report des amerikanischen Senats ${ }^{20}$.

20 Vgl. insgesamt zu diesem Komplex u. a. den Bericht in Der Spiegel Nr. 26/1977, S. 106 f.: „Souveränitä ist wichtiger als Folter". - W. Grabendorff: Die Lateinamerikapolitik der Regierung Carter, Arbeitspapier der Stiftung Wissenschaft und Politik AP 2131 vom April 1977. - Jetzt auch ders.: Das interamerikanische Verhältnis seit dem Amtsantritt Carters, in: Aus Politik u. Zeitgeschichte B 33-34/77 v. 20. 8. 1977, S. 29, 38. Zum Solidarisierungseffekt auf die linke Opposition mit der Militärregierung in Brasilien vgl. den Bericht in IHT vom 8. 3. 1977, S. 3: "Brazil opposition spurns U. S. rights view.“ 
Erosion der „Westlichen Hemisphäre“: Diversifizierung der Außenbeziehungen Lateinamerikas

Der Prozeß der Erosion des „Westliche Hemisphäre“-Konzeptes oder die Emanzipation Lateinamerikas von den USA wird besonders sichtbar — und bezieht zugleich von hier seine wichtigsten Impulse - in der Diversifizierung der politischen und wirtschaftlichen Beziehungen Lateinamerikas und in seiner wirtschaftlichen Entwicklung der letzten Jahre. Diese Offnung und Umorientierung Lateinamerikas wurde mit dem Mißerfolg der US-amerikanischen (und versuchten kollektiven interamerikanischen) Kuba-Politik eingeleitet. M. a. W. sie ging auf die mehr indirekten Wirkungen der kubanischen Entwicklungen auf die anderen lateinamerikanischen Staaten zurück. Die politische und wirtschaftliche Isolierung Kubas hatte - 1. das Castro-Regime schwächen, wenn möglich stürzen - 2. das kubanische Modell, den Kommunismus und Kubas Bindungen an ihn in Lateinamerika politisch und wirtschaftlich diskreditieren - 3. dadurch ein containment des Castroismus in Lateinamerika erreichen und - 4. dessen Stützung für die Sowjetunion so verteuern sollen, daß sie Kuba fallenlassen würde ${ }^{21}$. Die Punkte 1 und 4 waren völlige Mißerfolge, die Ziele 2 und 3 sind in etwa realisiert, wenn auch vermutlich ganz unabhängig von der amerikanischen Politik. Bleibende Wirkung für Lateinamerika hat weniger das konkrete kubanische Modell per se, sondern sein Symbolwert für die Möglichkeit einer von den USA unabhängigen Politik.

Es gibt mithin, wie sich damit gezeigt hat, für Lateinamerika Alternativen zur ausschließlichen US-Orientierung. Lateinamerika begann den Prozeß der politischen (und wirtschaftlichen) Diversifizierung zunächst bei sich selbst und entdeckte die Vielfalt möglicher Beziehungen zwischen seinen Einheiten und Subregionen, die früher im wesentlichen über Washington gelaufen waren. Dazu trägt nicht zuletzt die Verbesserung der Kommunikationsmöglichkeiten bei. Darüber hinaus gibt es Ansätze zur Schaffung intra-lateinamerikanischer auch politischer, vor allem aber wirtschaftlicher (über die letzteren sogleich) Assoziationen, Institutionen, Organisationen. Vor einigen Jahren war noch von der Anlehnung an Spanien und Portugal die Rede. Das ist vergessen. Es geht heute um das lateinamerikaninische (im Gegensatz zum interamerikanischen) System, einschließlich Kubas, aber ohne die Vereinigten Staaten.

\section{Erste und Zweite Welt}

Lateinamerika verbreiterte sodann die Basis seiner Beziehungen zum industrialisierten "Norden“ außerhalb der USA. Es suchte politische Beziehungen und Unterstützung, Handelspartner, Investoren, Partner für neuartige „joint ventures“, Kreditgeber, Lieferanten moderner Technologien (keineswegs alle so spektakulärer Art wie im Nuklearvertrag Brasilien-Bundesrepublik Deutschland) und Waffen.

Die Restriktionsbemühungen der USA in den beiden letztgenannten Bereichen waren wirkungslos bis konterproduktiv. Die USA hatten Lateinamerika seit längerem besonders teure und moderne Waffensysteme verweigert und sich unter Carter bemüht, darüber hinaus militärische Lieferungen jeglicher Art einzuschränken, um

21 Vgl. K. Krakau: Die kubanische Revolution und die Monroe-Doktrin (Frankfurt, Berlin 1968). 
das allgemeine Wettrüsten in Lateinamerika einzudämmen². Nun ist der finanzielle Aufwand für militärische Zwecke in Lateinamerika als Anteil vom BSP der geringste aller Weltregionen, und auch der Pro-Kopf-Aufwand liegt am unteren Ende der Skala ${ }^{23}$. Das mag immer noch viel sein für eine Region, die praktisch, von kleineren Grenzrivalitäten abgesehen, keine äußeren Bedrohungen kennt und für die der Militärapparat vor allem Prestige-Frage, Status-Symbol und Instrument für interne $\mathrm{Zwecke}$ ist: Repression, aber neuerdings auch, nicht unbedingt statt ihrer, sondern eher daneben: Modernisierung und Entwicklung. Gleichwohl ist für Lateinamerika die Haltung der USA einerseits Anlaß, sich erneut über die "Souveränitätsverletzung" durch die USA zu beklagen und sich im übrigen die gewünschten Waffen in Frankreich (Mirage-Düsenflugzeuge), England und Rußland (Panzer) und im übrigen Europa (BRD - gepanzerte Fahrzeuge, Schiffe) und Kanada zu beschaffen. Auch die amerikanischen Pressionen gegen den deutschbrasilianischen Nuklear-Vertrag ${ }^{24}$ verstärken diesen Trend, sind also für die USA konterproduktiv. Lateinamerika interpretierte sie als Versuche, den Subkontinent weiterhin in technologischer Abhängigkeit zu halten und die aufstrebende Großmacht Brasilien zu diskriminieren. Als solche wird Brasilien in Lateinamerika zwar sonst gefürchtet, doch hat sich Lateinamerika in diesem Zusammenhang sofort mit ihm gegen den Norden solidarisiert.

Über diese beiden speziellen Komplexe hinaus konnte Lateinamerika seine politischen sowie die Beziehungen in den anderen erwähnten wirtschaftlichen Bereichen "diversifizieren“, dadurch das Verhältnis zu den USA in einen größeren $\mathrm{Zu}$ sammenhang einbetten und zugleich das ideologische Solidaritätskonzept der "Westlichen Hemisphäre" schwächen. Es fand die gesuchten Partner zunächst in der Ersten Welt: Westeuropa (Frankreich, Spanien, Bundesrepublik), sodann in Kanada und Japan. Lateinamerika wandte sich insbesondere an wirtschaftsstarke Mittelmächte, die aber politisch schwach genug waren, um es politisch nicht zu gefährden. Aber es dehnte seine Suche schließlich auch auf die Zweite Welt der sozialistischen und kommunistischen Staaten Osteuropas, insbesondere die Sowjetunion aus. Die noch Anfang der 1960er Jahre mit ängstlichem Blick auf Washington vermiedenen diplomatischen Beziehungen zu Osteuropa und China wurden inzwischen allgemein aufgenommen. Auch die früher automatische Abstimmungssolidarität Lateinamerikas mit den Vereinigten Staaten in der UNO existiert schon lange nicht mehr.

Der Nutzeffekt dieser neuen erweiterten Beziehungen ist zwar nicht der eines Wunderheilmittels für die Probleme der Wirtschaftsentwicklung und Abhängigkeit Lateinamerikas. Sie bringen auch neue Probleme mit sich. Politisch - eine Zeitlang gerieten einige lateinamerikanischen Staaten, nicht nur Kuba, in die ideologischen und revolutionsstrategischen Auseinandersetzungen zwischen Moskau und Peking; das hatte Auswirkungen auf die innenpolitische Situation in diesen Ländern. Wirtschaftlich - für Lateinamerika ergeben sich Nachteile aus den Präferenzabkommen der EG mit Afrika und Asien, die Lateinamerika ausschließen. Aber diese Beziehungen wirken doch wegen ihrer Vielgestaltigkeit untereinander und insgesamt im Verhältnis zu den USA komplementär. Sie haben für

22 Carters Lateinamerika-Rede oben Anm. 15; ferner ders. im Mai, vgl. USIS Official Text vom 23. 5. 1977.

23 Daten u. Tabellen bei James D. Theberge u. Roger W. Fontaine (eds.): Latin America: struggle for progress. Critical Choices for Americans XIV (Lexington, Toronto 1977), S. $24 \mathrm{f}$.

$24 \mathrm{Vgl}$. schon Norman Gall: Atoms for Brazil, danger for all, in: Foreign Policy 23 (Summer 1976) S. 155-201. 
Lateinamerika außerhalb der USA neue Importquellen für die Lieferung von Waren und Industriegütern (allein aus Japan 1974 ein Anteil von über 10 Prozent), von Kapital (Investitionen, mit besonders hohem Anteil Japans, vor allem in der industriellen Verarbeitung, nicht mehr in den klassischen Extraktionsbereichen; in diesen hat man erfolgreich mit joint ventures experimentiert) und technologischem Wissen, daneben aber auch Absatzmärkte für die eigenen Exporte (Rohstoffe, Nahrungsmittel, aber auch zunehmend Industrieerzeugnisse) erschlossen (der Anteil Westeuropas beträgt in beiden Richtungen etwa 30 Prozent). Auch die sozialistischen Staaten, am geringsten unter ihnen China, haben dazu beigetragen. Der Schwerpunkt ihrer wirtschaftlichen Aktivitäten liegt, von Kuba abgesehen, in Brasilien, Argentinien, Peru, Kolumbien ${ }^{25}$. Während 1950 noch fast 50 Prozent aller lateinamerianischen Exporte in die USA gingen und der Anteil der lateinamerikanischen Importe aus den USA 57 Prozent betrug, sind die entsprechenden Anteile 25 Jahre später nur noch 32 und 37 Prozent. Japanische Investitionen in Brasilien überstiegen 1973 erstmals diejenigen der USA.

\section{Dritte Welt}

Schließlich dehnt Lateinamerika den Bezugsrahmen für sein Verhältnis zu den USA noch weiter auf dasjenige der 3. Welt zu den Industriestaaten schlechthin aus und sieht sich als Teil der 3. Welt im allgemeinen Nord-Süd-Konflikt den Vereinigten Staaten gegenüber. Dabei sucht Lateinamerika ebensosehr die seinen ökonomischen Problemen angemessene wirtschaftspolitische Zuordnung und Verbesserung der entsprechenden Verhandlungspositionen wie allgemein die Stärkung seiner politischen Unabhängigkeit und Handlungsfreiheit gegenüber den USA.

Diese neue Komponente der Außen- und internationalen Orientierung Lateinamerikas enthält eine ganze Reihe von Widersprüchen und Problemen. Was konstituiert letztlich die „3. Welt“ als politische Bewegung, Kraft, Staatengruppe, Block o. ä.? Gehören die Staaten Lateinamerikas überhaupt dazu? Was haben sie mit denen Afrikas und Asiens gemeinsam? Soweit das anfängliche politische Ziel der 3. Welt die wirkliche und vollständige Unabhängigkeit und Entkolonialisierung war, steht die lange Tradition staatlicher Unabhängigkeit Lateinamerikas dagegen. Der neutralistischen Konzeption (non-alignment) einer ungebundenen Staatengruppe zwischen den Blöcken widerspricht die Bindung an die USA in Rio-Vertrag und OASCharter, die etwa freiwillig zu verlassen noch keinem Staate - nicht einmal Kuba - eingefallen ist; ferner steht $\mathrm{zu}$ ihr die historische, politische, kulturelle Bindung an Europa und trotz allem auch an die USA im Widerspruch. Lateinamerika konnte im Grunde erst zur 3. Welt stoßen, als die Probleme der wirtschaftlichen Entwicklung/Unterentwicklung, Abhängigkeit und deren Überwindung sowie das Ziel der Errichtung einer gerechteren neuen Weltwirtschaftsordnung in den Vordergrund des Interesses traten (Lusaka 1970). Obwohl mehrere lateinamerikanische Staaten zu den am meisten entwickelten in dieser Gruppe zählen, übernahm Lateinamerika alsbald eine führende Rolle in dieser Bewegung: in der Entwicklung theoretischer Konzepte zur Analyse der Unterentwicklung (dependencia-Theorie); in der darauf fußenden Formulierung praktischer wirtschaftlicher und politischer

25 Vgl. die Angaben und Tabellen bei Theberge und Fontaine a. a. O. S. 5-14. 
Forderungen und Strategien zu ihrer Überwindung - hier ist der jahrelange bedeutende Einfluß R. Prebischs über die ECLA auf die gesamte 3. Welt zu nennen; schließlich in der politisch-organisatorischen Wirksamkeit bei der Errichtung neuer Organisationen und Institutionen zur Artikulierung und Realisierung dieser Strategien, Forderungen, Programme, Interessen. Vertreter lateinamerikanischer Staaten waren maßgeblich beteiligt an der Konstituierung der "Gruppe der 77" auf der UNCTAD 1964, an der Gründung von OPEC, CIPEC (Kupfer) und IBA (Bauxit), der Propagierung und schließlichen Verabschiedung der "Charter of the Economic Rights and Duties of States", der Konzeptualisierung des New International Economic Order, auf der UN-Sonderkonferenz 1974 (Mexiko, wenngleich mit deutlichem Blick auf die mexikanische Innenpolitik ${ }^{26}$, an der Abhaltung der Pariser Nord-Süd-Konferenz CIEC (Venezuela als Co-Chairman) usw. ${ }^{27}$.

Vertreter Lateinamerikas gehören zu den wichtigsten Sprechern, die Staaten der Region zu den stärksten Motoren in dieser Bewegung, die es sich zum Ziel gesetzt hat, durch Verhandlungen, Pressionen, notfalls Konfrontationen eine neue, bessere, gerechtere $-d$. h. die Interessen der weniger entwickelten Staaten mehr als bisher berücksichtigende - Weltwirtschaftsordnung zu schaffen. Der starke politische wie auch moralische Impuls dieser Bewegung beruht darauf, daß sie die Unterentwicklung als ein notwendiges Produkt der Entwicklung der Industriestaaten interpretiert und darüber hinaus seit dem Bericht von Rom über die „Grenzen des Wachstums" mit dem Vorwurf des selbstzerstörerischen Überkonsums in den Industriestaaten deren Modellcharakter grundsätzlich in Frage stellt, zugleich aber die eigene neue und höhere Moral der 3. Welt postuliert. Die Zuordnung zur 3. Welt löst natürlich für sich noch nicht die Wirtschaftsprobleme Lateinamerikas oder der übrigen 3. oder gar der „4."Welt. Sie dient deshalb auch gar nicht eigentlich der Intensivierung wirtschaftlicher Austauschbeziehungen mit ihr. Diese Beziehungen werden zwar mit zunehmender Industrialisierung auch expandieren, wenn auch, noch lange marginal bleiben. Aber die Zuordnung bewirkt insgesamt dreierlei:

1. Sie führt zu einer Lockerung der wirtschaftlichen und politischen Bindungen und Abhängigkeiten Lateinamerikas von den bzw. an die USA; die Diversifizierung dieser Beziehungen und die Bildung größerer Gruppierungen mit gemeinsamen Interessen, die den USA gegenüberstehen, stärkt die Selbständigkeit und Souveränität der lateinamerikanischen Staaten, ermöglicht ein erhöhtes Maß an Solidarität unter ihnen und trägt zum Abbau der Fremdbestimmung bei.

In diesem Zusammenhang wäre darauf hinzuweisen, daß US-amerikanische Maßnahmen gegen einzelne Lateinamerikanische Staaten zunehmend solidarische Reaktionen aller lateinamerikanischen oder aller 3.-Welt-Staaten auslösen. Der Trade Act 1974 enthält zwar einerseits ein liberalisiertes allgemeines Präferenzsystem, nahm davon aber strafweise die OPEC-Länder Venezuela und Ekuador wegen der Ölpreiserhöhungen aus. Die Proteste Lateinamerikas waren vehement und solidarisch (der Ständige Rat der OAS verurteilte sie als „diskriminierende Zwangsmaßnahme“). Die Gründe sind einmal die naheliegenden politischen. Aber auch die Öl-

26 Vgl. Wolf Grabendorff: Die Außenpolitik Mexikos und ihre innenpolitischen Ziele, in: Berichte zur Entwicklung in Spanien, Portugal und Lateinamerika (Nov./Dez. 1976), S. 40-48.

$27 \mathrm{Vgl}$. näher zum ambivalenten Verhältnis Lateinamerikas zur 3. Welt: Theberge u. Fontaine a. a. O. S. 15 ff.; Manfred Nitsch: Latin America and the Third World, in: Vierteljahresberichte, Probleme der Entwicklungsländer, Forschungsinst. der Friedr.-Ebert-Stiftung Nr. 68 (Juni 1977) S. 91-105; H. C. F. Mansilla: Latin America and the Third World: similarities and differences in development concepts, ibid. S. 119-135. 
importierenden, also von den höheren Preisen betroffenen Länder protestierten im Hinblick auf die Präzedenzwirkung der amerikanischen Regelung für etwaige spätere weitere Rohstoff-Kartell-Abkommen, und Rohstoffproduzenten sind praktisch alle Staaten Lateinamerikas. Außerdem sieht das Gesetz Anpassungen der Präferenzen im Hinblick auf einzelne Länder durch den US-Präsidenten vor - ein zusätzliches Instrument in seiner Hand zur Disziplinierung politischer oder wirtschaftspolitischer Dissidenten in Amerika, gegen das man sich antizipatorisch wehren möchte.

2. Die Gruppenbildung mit der 3. Welt könnte (zumindest ist das die Hoffnung aller) die gemeinsame wirtschaftliche Schwäche in politische - und moralische? Verhandlungs-Stärke umwandeln: zunächst im Hinblick auf das globale Ziel einer gerechteren neuen Weltwirtschaftsordnung im Verhältnis zu den Industriestaaten schlechthin, sodann aber auch konkreter im Verhältnis Lateinamerikas zu den USA. Die Lösung der lateinamerikanischen Wirtschaftsprobleme erhält dadurch eine neue Perspektive: sie wird von der Ebene der traditionellen bilateralen Verhandlungen mit den USA über Kapitalhilfe - möglichst zu Vorzugsbedingungen - oder über Zuckerquoten usw. auf die multilaterale Ebene interamerikanischer (z. B. InterAmerikanische Entwicklungsbank) oder mondialer Institutionen (Weltbank, IMF, CIEC u. a.) verlagert. Lateinamerika wird zwar noch lange Rohstoffe exportieren und Kapital importieren. Aber die einzelstaatlichen Probleme werden eingebettet in den gesamtamerikanischen oder noch allgemeiner in den Nord-Süd-Dialog über allseitige Zollpräferenzen, terms of trade, Rohstoff-Fonds und -stabilisierungsabkommen usw. - kurz, über den angemessenen Zugang zum Markt der Industriestaaten und über die Stabilisierung des internationalen Marktes.

3. Schließlich bilden diese beiden Zielsetzungen in Lateinamerika einen politischen Bezugsrahmen, in den sich alle Staaten der Subregion ohne Ansehen ihrer innenpolitischen Orientierung widerspruchslos einordnen können, von liberal-demokratischen über progressiv-reformerische und konservativ-entwicklungsdiktatorische bis zu reaktionär-traditionalistischen Regimen. Brasilien und Peru, Mexiko, Chile und Paraguay können sich in diesem Rahmen solidarisch fühlen ${ }^{28}$.

\section{Interne Wirkungen: Ausweitung der Staatsfunktionen}

Noch ein weiteres Moment verbindet die unterschiedlichsten Regimes innerhalb Lateinamerikas (und der 3. Welt allgemein) zu einer einheitlichen Position und stärkt sie gegenüber den USA: die Betonung der überragenden Rolle des Staates. Hier verbindet sich die ältere politische 3. Welt-nonalignment-Bewegung (äußere staatliche Selbständigkeit) mit der jüngeren ökonomischen Variante: der Staat wird inzwischen übereinstimmend als wichtigster unmittelbar handlungsfähiger und verantwortlicher Akteur im wirtschaftlich-politischen Entwicklungsprozeß angesehen: ein Neo-Merkantilismus ${ }^{29}$. Der staatliche Sektor ist überall in Lateinamerika erheblich gewachsen (in Brasilien umfassen Staatsausgaben 37 Prozent des

\footnotetext{
28 Allgemein hierzu auch W. Grabendorff: Lateinamerikas Rolle in der Weltpolitik, in: Berichte ... (März/April 1976), S. 27-32; speziell zur heutigen. Rolle des Militärs in diesen Prozessen in Lateinamerika ders.: Militärherrschaft in Lateinamerika, ibid. (Mai/Juni 1976) S. 23-38, ferner die Beiträge von Catterberg, Zamora und Castillo zum Thema "Militarismo y Desarrollo", in: Josef Thesing (Hrsg.): Politica y Desarrollo en America Latina (Bonn, 1976?) S. $165 \mathrm{ff}$.

$29 \mathrm{Vgl}$. Nitsch a. a. O. S. 96 f.; Mansilla a. a. O. S. 128 f.
} 
BSP; in Peru macht der staatliche Anteil über 50 Prozent der Gesamtinvestitionen aus). Dieser Einstellung kommt die Bereitschaft nichtamerikanischer Kapitalgeber $\mathrm{zu}$ sog. joint ventures entgegen. Auf der lateinamerikanischen Seite kommt diese Position in der Welle von Verstaatlichungen ausländischen, $d$. h. insbesondere nordamerikanischen, Eigentums zum Ausdruck, auf welche die USA trotz sehr unterschiedlicher Regelung der Entschädigungsfrage (Kuba, Venezuela, Peru) aber inzwischen gelassen reagieren ${ }^{30}$. Aus Bereichen wie Öl, Petrochemie, Eisen, Kupfer (Venezuela, Chile mit und nach Allende), in denen die USA früher ein Monopol hatten, sind sie fast oder vollständig verdrängt. Linke bis rechte - staatssozialistische und staatskapitalistische - Regime treffen sich hier in der konservativ- oder entwicklungsprogressiv-) diktatorischen Behauptung der Staatsgewalt nach innen und der Zurückdrängung des nordamerikanischen wirtschaftlichen Einflusses nach außen - Stärkung der zugleich den internen Konsens fördernden und alle Gruppen integrierenden Souveränität. Auch in der Abwehr der von außen kommenden Anfechtungen solcher etatischer Maßnahmen kann man sich auf alte lateinamerikanische Tradition - die Calvo-Doktrin ${ }^{31}$ stützen, deren Grundkonzeption in der Dritten und, halb gezwungen, in der Ersten Welt in der Economic Charter von 1974 (II. 2.) übernommen worden ist. Die staatliche Kontrolle/Steuerung der Volkswirtschaft dehnt sich im selben Maße aus wie der Freiraum für free enterprise American style schrumpft. Und die USA haben gelernt, damit zu leben.

\section{Rückbildung des Monroe-Reflexes}

Diese internen Veränderungen und die mit ihnen einhergehenden internationalen Umorientierungen und erweiterten Beziehungen Lateinamerikas zur Ersten, Zweiten und Dritten Welt gelten aber inzwischen auch für die USA nicht mehr unbedingt als "extrakontinentale Intervention" oder "Assoziation" und lösen nicht mehr den Monroe-Reflex aus. Diese entspanntere Haltung der USA wurde möglich, weil das internationale System seine bipolare Starre und die Vereinigten Staaten die unmittelbare Furcht vor dem weltpolitischen Gegner verloren haben und weil sie deshalb das Sicherheitsrisiko in Amerika entsprechend niedriger einschätzen. Konkreter ergab sich das aus den russisch-amerikanischen Folgevereinbarungen nach der Kuba-Krise einschließlich der Stützpunktabrede, nach den kubanischen Mißerfolgen in Bolivien wie andernorts in Lateinamerika (z. T. aus Finanzschwäche) sowie aus der wachsenden Abhängigkeit Kubas von der zur Mäßigung neigenden und an détente interessierten Sowjetunion. Aber davon ganz abgesehen - die USA können auch nicht mehr mit Aussicht auf Respektierung in Lateinamerika den Rahmen des international für Lateinamerika Erlaubten oder Verbindlichen definieren. Dazu sind die angedeuteten Entwicklungen zu weit fortgeschritten. Gleichwohl käme der eigentliche Test für eine tolerante Haltung der USA natürlich erst dann, wenn sich die außenpolitische Distanzierung eines Staates in Lateinamerika mit revolutionären Veränderungen im Innern verbindet (wofür z. Z. keine Anzeichen sichtbar sind). Würde ein solcher Vorgang nicht mehr als „unzu-

30 Dazu Cole Blasier: The hovering giant. U. S. responses to revolutionary change in Latin America (Pittsburgh 1976), bes. Kap. 8 S. $241 \mathrm{ff}$.

31 Vgl. dazu Knud Krakau: Lateinamerikan. Doktrinen zur Realisierung staatlicher Unabhängigkeit und Integrität, in: VRƯ 8 (1975) S. 117-144, bes. $119 \mathrm{ff}$. 
lässiges" Überwechseln ins andere „Lager" gewertet werden? Ob die amerikanischen Versuche, mit Kuba zu einem modus vivendi zu kommen, als positives Zeichen dafür zu werten sind, erscheint durchaus fraglich, denn schließlich fügen die USA sich nur, fast 20 Jahre post factum, in das Unabänderliche. Ähnliches gilt für die grundsätzliche Einigung über einen neuen Kanalvertrag mit Panama ${ }^{32}$.

\section{Der intraregionale Beitrag}

Der als Folge der Veränderungen im interamerikanischen und internationalen System eingeleitete Emanzipationsprozeß Lateinamerikas wird durch einige bedeutende Wandlungen unterstützt, die in den letzten Jahren in der wirtschaftlichen Lage Lateinamerikas, jedenfalls in einer ganzen Reihe von Staaten der Region und ihren Beziehungen zu den USA eingetreten sind. Sicher ist der Höhepunkt der nordamerikanischen Dominanz in den Wirtschaften Lateinamerikas seit längerem überschritten, wie er auch schon in den frühen 50er Jahren vor der kubanischen Revolution in Kuba überschritten war. Anzeichen dafür ist nicht zuletzt die verbesserte Leistungsfähigkeit der lateinamerikanischen Wirtschaften auf praktisch allen Gebieten, so daß sich die Abhängigkeit von den USA zumindest relativ vermindert. Das allgemeine Wirtschaftswachstum hatte sich bis 1974 auf etwa 6 Prozent p. a. erhöht (Brasilien gut 10 Prozent ïber fast 10 Jahre), fiel dann allerdings infolge der Energieverteuerung und nachfolgenden Rezession wieder auf unter 3 Prozent. Aber die Nahrungsmittelproduktion steigerte sich, die Bevölkerungsexplosion konnte gebremst, die Exporterlöse konnten gesteigert, die terms of trade insgesamt, nicht zuletzt als Folge der zuvor beschriebenen Diversifizierung, und die allgemeinen Infrastrukturen der meisten Länder verbessert werden ${ }^{33}$.

Umgekehrt ist die nordamerikanische Abhängigkeit von einigen strategisch wichtigen und nicht substituierbaren Rohstoffen aus Lateinamerika relativ größer geworden. So kommt das für Düsentriebwerke benötigte Niobium in den USA selbst überhaupt nicht vor; sie beziehen über 50 Prozent ihres Bedarfes aus Brasilien. Bauxit hat eine ähnliche Bedeutung für Bolivien und Jamaica und die USA. Nun spielt dieses Argument bekanntlich in der Imperialismus-Diskussion neben dem Markt- und Investitionstopos eine bedeutende Rolle. In dem Maße aber, in welchem die lateinamerikanischen Staaten den USA nicht mehr isoliert und auch nicht mehr nur als Subregion gegenüberstehen, sondern als Glieder größerer Einheiten, kann sich die frühere Verwundbarkeit in verhandelbare Selbständigkeit umkehren.

Hier liegt die Bedeutung der oben skizzierten Zuordnung Lateinamerikas zur 3. Welt. In diesem Zusammenhang ist aber auch auf die regionalen und subregionalen wirtschaftlichen Zusammenschlüsse, Koordinations- und Kooperationsmechanismen in Lateinamerika hinzuweisen. Sie können hier lediglich erwähnt werden: die allgemeine Lateinamerikanische Freihandelszone und der aus ihr hervorgegangene, aber zumindest anfangs um so effizientere Anden-Pakt (Gemeinsamer Markt), die Gemeinsamen Märkte in Zentralamerika und in der Karibik. Die Zielsetzungen waren im Grunde stets dieselben: Schaffung größerer Märkte durch Abbau der Bin-

\footnotetext{
32 Bericht darüber in USIS News Report vom 13. 8. 1977.

33 Vgl. z. B. dazu Theberge u. Fontaine a. a. O. S. 16 ff. (etwas pessimistischer); A. Lowenthal a. a. O. S. $206-8$.
} 
nen- und Errichtung gemeinsamer oder wenigstens Koordinierung der Außenzölle und dadurch Stimulierung der eigenen Produkte, der landwirtschaftlichen und insbesondere der industriellen, großräumige gemeinsame Planung der wirtschaftlichen Entwicklung, der Industrialisierung, der Investitionen, Regulierung ausländischer Investitionen und des Verhaltens der internationalen Konzerne usw. Auch das Schicksal dieser Bemühungen war im allgemeinen gleich: Rückschläge und Scheitern nach hoffnungsvollen Anfangserfolgen (besonders in der Andenregion) infolge der wirtschaftlichen, aber auch politischen Probleme (Rivalitäten, Regimewechsel usw.). Ein neuer Ansatz ist das 1975 entstandene Lateinamerikanische Wirtschaftssystem (SELA). Es versucht, die Impulse der Dritten-Welt-Bewegung auszunutzen und innerhalb Lateinamerikas - einschließlich Kubas, ohne die USA - die wirtschaftliche Kooperation zu fördern und die wirtschaftlich-sozio-politischen Positionen der Staaten der Region $\mathrm{zu}$ koordinieren und $\mathrm{zu}$ gemeinsamen Positionen und Strategien gegenüber der Außenwelt - den USA, dem "Norden“ allgemein zu aggregieren. Man akzeptiert, daß die einzige wirkliche Gemeinsamkeit die allgemeine Unterentwicklung und die ungleiche Teilhabe an der Weltwirtschaft ist. Man respektiert die intraregionale Heterogenität und staatliche Souveränität und sucht mit einem möglichst unaufwendigen institutionellen Überbau auszukommen ${ }^{34}$. Dieser Ansatz ist zugleich zurückhaltender und ambitiöser als seine Vorgänger. Seine Wirksamkeit bleibt abzuwarten.

\section{Schlußbemerkung: Evaluierung}

Die dargestellten Entwicklungen haben in relativ kurzer Zeit - seit etwa der zweiten Hälfte der 1960er Jahre - eine erstaunliche Veränderung des Selbstbewußtseins und der politischen Attitüde der Staaten Lateinamerikas gegenüber den USA und anderen Regionen hervorgebracht. Bis dahin hatten Entwicklungen oder Ereignisse der dargestellten Art sie immer wieder zu nationalistischen Ausbrüchen veranlaßt, gefolgt von Klagen über die „Vernachlässigung“ durch Washington, d. h. aber zur hilflosen und alternativlosen Anlehnung an die USA. Inzwischen scheint der spell der „Westliche-Hemisphäre“-Ideologie wie eine Wolke verflogen zu sein. Man entdeckt verwundert, daß die amerikanische Geographie keine wirtschaftliche, politische, ideologische Einheit stiftet, daß das Konzept der „Westlichen Hemisphäre" bestenfalls eine Fiktion ist, daß die Interessengegensätze im hier sinnfälligen Nord-Süd-Konflikt oft größer als manche fortbestehenden Übereinstimmungen sind. Lateinamerika ist in vieler Hinsicht "freier" als es je war - sicher nicht befreit von seinen langfristigen wirtschaftlichen strukturellen Problemen, aber befreit von der bisherigen Automatik-Bindung in allen Lebensbereichen an die USA und insofern „frei“, diese Probleme in sachorientierten $\mathrm{Zu}$ sammenhängen anzugehen, die in höherem Maße als bisher selbst gewählt werden können.

34 Die Literatur zur Integrationsproblematik ist unübersehbar, vgl. z. B. Félix Peña: Perspektiven der wirtschaftlichen Integration Lateinamerikas, in: Wolf Grabendorff (Hrsg.): Lateinamerika - Kontinent in der Krise (Hamburg 1973) S. 299-314; Jaime Campos: Problemas económicos y polítícos de la integración latinoamericana, in: J. Thesing (ed.): Política y desarrollo a. a. O. S. 67-82; vor allem von einem der besten Kenner der Materie, Miguel S. Wionczek, (außer verschiedenen Büchern) die vorzügliche neuere Analyse: Latin American growth, trade and cooperation, in: Vierteljahresberichte, Probleme der Entwicklungsländer, Friedr.-Ebert-Stiftung Nr. 68 (Juni 1977), S. 107-118, speziell zum SELA S. $116-118$. 
Dabei ist es eine Ironie, daß diese Emanzipation in Lateinamerika mit wenig Ausnahmen von konservativen und einigen reformistisch-progressiven Militärdiktaturen vollzogen wird. Noch 10 Jahre zuvor schien diese Aufgabe demokratisch-liberalen Regimen vorbehalten. Mit den Militärregimen verbindet die USA ideologisch wenig. Strategisch hat Lateinamerika ohnehin seine Bedeutung weitgehend verloren und ihre Rohstoffinteressen suchen auch die Vereinigten Staaten eher global im Nord-Süd-Dialog zu befriedigen.

Die Regierung Carter hat die wichtigsten Veränderungen in Lateinamerika - den Zerfall des einheitstiftenden ideologischen Konzeptes der "Westlichen Hemisphäre“ und die Zuordnung Lateinamerikas zur 3. Welt - registriert und offenbar akzeptiert. Sie vermeidet die große ideologische „community“-Rhetorik und erkennt statt dessen den "global charakter of the region's problems" (Carter) an. Sie sucht keine regionalspezifischen Programme oder Lösungen mehr, keine HemisphärenIdeologie-befrachtete Fortsetzung von Sonderpolitik in der Reihe der Good Neighborhood, Alliance for Progress, New Dialogue (Kissinger) und ähnlicher Schlagworte. Auch die Menschenrechtspolitik ist ja keineswegs Amerika-spezifisch. Die Carter-Regierung nimmt vielmehr Lateinamerika beim Wort: auch sie interpretiert die Handels- und Wirtschaftsprobleme der Region im globalen Kontext und kündigt Beiträge zu ihrer Lösung lediglich im Rahmen des allgemeinen NordSüd-Dialoges oder-Konfliktes an ${ }^{35}$.

So besteht vielleicht zum ersten Mal die Chance, daß sich die Beziehungen der USA zu Lateinamerika distanziert-normal entwickeln - nicht etwa konfliktfrei, das wäre das alte verworfene Harmonie-Konzept. Gegenstand des Nord-Süd-Konfliktes ist Umverteilung von Gütern, Chancen und Reichtümern, aber eine Konfliktaustragung nach definierbaren Interessen und Sachgesichtspunkten statt nach der Geographie und einer realitätsfernen Ideologie.

\footnotetext{
$35 \mathrm{Vgl}$. hierzu insbesondere die schon (Anm. 15) zit. Rede Carters zur Lateinamerika-Politik; danach haben die USA drei Prioritäten: 1. ,high regard“ für die individuelle Souveränität; 2. human rights; 3. Anerkennung des globalen Charakters der wirtschaftlichen Probleme Lateinamerikas, die identisch mit dem Problem d. Beziehungen USA-Entwicklungsländer allgemein seien und nicht "solely on regional terms", behandelt werden könnten. Ferner die wichtige Rede vom 22. 5. 77 vor der Notre Dame University, USIS Official Text vom 23. 5. 77 bs. S. 6. - Immerhin betonte auch schon Kissinger trotz seiner überzogenen Rhetorik die Einbettung des "traditional special relationship “ in den globalen Zusammenhang vor allem der Nord-Süd-Beziehung, Rede am 6. 7. 1976 vor dem Chicago Council on Foreign Relations, USIS Official Text vom 7. 7. 76, bes. S. 9. Dieses bedeutende Dokument ist eine Art summa der praktischen Politik Kissingers (Anlaß: Bicentennial). Vgl. ferner Lowenthal a. a. O.; Wolf Grabendorff: Außenpolitische Emanzipation Lateinamerikas, in: ders. (Hrsg.) Lateinamerika a. a. O., S. 340-378; ders. schon Mai 1972, „Aufzeichnung betr. Die Emanzipation Lateinamerikas: Ursachen und außenpolitische Folgen", Stiftung Wissenschaft und Politik (AZ 1082 Fo. Pl. IV. 3/72); zuletzt ders.: Lateinamerika und die Vereinigten Staaten, in: Europa Archiv (1977) S. 433-440, und ders.: Das interamerikanische Verhältnis (oben Anm. 20).
} 


\title{
Fundamental Rights and the Nigerian Draft Constitution
}

\author{
By A. S. Fadlalla
}

Under the 1963 Constitution, Nigeria had an impressive list of Fundamental Rights modelled on the European Convention of Human Rights. Those guarantees, with some modifications, became the basis for the New Draft. In the past, these guarantees were criticised for being "liberal" rights that make no reference to the social or economic rights of the individual; that they were merely "declaratory" of existing law and that they were so hedged with exceptions as to render them ineffective in practice. Moreover, the Judiciary was criticised for adopting a too cautious attitude in interpreting those guarantees. None of these criticisms, however, need derogate substantically from their effectiveness.

The New Draft shows many marked improvements. The drafting is more neat and for once the emphasis is first placed on the rights protected, followed by the recognised exceptions, many of which had been removed. However, it is still possible to limit most of the rights by a law which is "reasonably justifiable in a democratic society" an elusive phrase which has not been clearly defined by the courts. Needless to say that a lot would still depend on the attitude of the courts, and it is suggested that they might be helped in this task if the word "necessary" should be substituted for "reasonably justifiable" as something could be reasonably justifiable without being necessary.

The paper also points out certain issues of controversy pertaining to freedom of the Press and freedom from discrimination. And finally it draws attention to some other areas where possible problems might arise in future, notably, the likely conflict between the Bill of Rights and the list of Directive Principles e. g. by insisting on the entrenchment of the right to property and the payment of adequate compensation for any interference with it, the Bill may have made it difficult for any future government bent on securing the "maximum welfare" of every Nigerian citizen so to act.

\section{The United States and Latin America}

\section{By KNUd KRAKAU}

In the short run the Carter administration uses the by now familiar carrotand-stick tactics vis-à-vis Latin American governments: granting or with-holding foreign aid (military, economic), thereby rewarding or punishing the good or the bad governments, respectively - now in the interests of "human rights". In the past the good/bad guys were defined in terms of their attitudes towards fascism, "international communism", democracy, dictatorship, free elections, nationalization of foreign (American) property etc. But at the same time perspectives seem to change in a more fundamental way. For more than a century and a half US relations with Latin America have been troubled by the very "success" of the most hallowed US foreign policy taboo, the Monroe Doctrine. Thoug really no success at all or if, at least so for the wrong reasons, it later served as a convenient cloak for a hard-nosed hegemonial policy and misled the US until very recently into 
defining every problem arising in its relations with Latin America by way of automatic reflex in terms of the Monroe Doctrine instead of on its individual merits. Thus it severly limited US capacity of perception of the complexity of its relations with more than 20 very heterogeneous countries. But the new administrations seems to sense that there is little reason for any special "Western Hemisphere relationship" between the US and Latin America, that politico-economic conditions - underdevelopment and structural dependency - rather push the countries of Latin America into the "Third World". The Latins, even though improving on their economic performance, consciously try to act this new role, diversifying, with partial success, not only their internal economies, but also their economic and political international relations, not. long ago centered exclusively on the US. The Carter administration has announced its readiness to respond to this reorientation and for some time American governments have quietly accepted Latin American nationalizations and the expanding role of the governmental sector coming with them. Carter now defines the problems with Latin America in terms of general North South relations. Though no immediate solution for them, at least - and at last - this is a realistic basis for their assessment. 